\title{
Low-Power Radio Communication in Industrial Outdoor Deployments: The Impact of Weather Conditions and ATEX-compliance
}

\author{
Carlo Alberto Boano ${ }^{1}$, James Brown ${ }^{2}$, Zhitao $\mathrm{He}^{1}$, \\ Utz Roedig $^{2}$, and Thiemo Voigt ${ }^{1}$ \\ 1 Swedish Institute of Computer Science, Kista, Sweden \\ \{cboano, zhitao, thiemo\}osics.se \\ 2 Lancaster University Computing Deparment, Lancaster, UK \\ $\{j$.brown, u.roedig\}@lancaster.ac.uk
}

\begin{abstract}
Industry recognizes wireless sensor networks as one of the next major technical and economical shifts in automation and control systems. Industrial applications need performance assurances of wireless communication, which is hard to attain given the variability of the environment where such applications are deployed. In this paper, we quantify the impact of environmental conditions, namely temperature, fog, and rain on wireless communication links. We further investigate the influence of industrial casing on communication, evaluating an ATEX enclosure intended for use in potentially explosive atmospheres. Knowledge of the potential impact of environmental conditions and special casings provides an important input to robust communication design during deployment planning.
\end{abstract}

Key words: Industrial outdoor deployment, wireless sensor networks, temperature impact, weather conditions, RSSI, LQI, ATEX compliance

\section{Introduction}

Several industry sectors are seeing wireless sensor networks as a promising technology to bring operational renovations. A wide range of potential applications, such as plant automation, process monitoring and control, preventive maintenance, safety in hazardous environments, and radiation detection, can benefit from the technology. Wireless sensor networks increase efficiency in automation and process control by enabling quicker decisions, improving safety and reliability, enhancing flexibility and visibility, while decreasing maintenance and installation costs.

Industrial applications, on the other hand, have strict requirements for delay and reliability. The required performance assurance is hard to attain without knowledge about the complicated variability of the environment where the sensors are deployed. Unlike military applications where nodes are placed in a dense and random manner, industrial plants allow carefully planned sensor deployments to facilitate the provision of performance assurances. For example, 
modern industrial plants such as refineries may host more than ten thousands sensors. In these plants, extensive redundancy is impossible due to the sheer numbers of sensors and the associated costs.

When deploying outdoor industrial sensor networks, persistent connectivity between sensors must be maintained over a range of environmental conditions. This is a major challenge, in light of the known impacts of temperature and weather conditions on sensor nodes and their low-power radios. Bannister et al. have observed a strong reduction in signal strength during the hottest time of the day [1], while others have attributed transmission range and connectivity changes to fog and rain [2, 3, 4].

Another outstanding issue that potentially impacts radio communication are the specific implementation requirements. For example, equipment for products intended for use in potentially explosive atmospheres must be ATEX certified [5]. While it would be possible to obtain ATEX certifications for sensor nodes, a simpler way is to put regular non-certified sensor nodes into ATEX-compliant enclosures. However, the potential impact of such enclosures on wireless communication has never been quantified to date.

In this paper, we analyze the impact of environmental conditions and certification requirements on wireless connectivity in industrial outdoor deployments. We also quantify these effects, in order to enable solutions that can give performance assurances to radio communication.

The contributions of our work are three-folded. Firstly, we experimentally confirm the findings by Bannister et al. [1] showing that increasing temperature negatively affects the communication between nodes. Further, we analyze the behavior of nodes below $0^{\circ} \mathrm{C}$. Secondly, we assess the impact of weather effects such as fog, rain, and snow on low-power radio communication. Thirdly, we measure if ATEX compliant enclosures affect the communication between sensor nodes.

The paper proceeds as follows. Section 2 provides a general description of the related work. We investigate and quantify the impact of temperature on radio communication in Section 3. Thereafter we discuss the influence of weather phenomena in Section 4, providing a comparison between the impacts of temperature, weather and radio interference. We quantify the impact of ATEX compliant enclosures on the communication between sensor nodes in Section 5. We conclude the paper in Section 6, along with a summary of the results and suggestions for future work.

\section{Related Work}

Several researchers have shown that outdoor sensor networks are affected by weather conditions and temperature. The results of Anastasi et al. suggest that weather effects, specifically fog and rain, may have a severe impact on the transmission range of sensor nodes [2]. They report a high difference in the maximum range between two MICA2 nodes: 70 meters with clear and dry weather conditions versus 25 meters with foggy and rainy conditions. The authors do not 
present any details about the amount of rain, humidity, and temperature. In their experiments, they provide a relationship between the Packet Reception Rate $(\mathrm{PRR})$ and the distance between nodes, without any allusion on the signal strength or link quality.

Sun et al. [3] report a loss of connectivity in their outdoor experiments with rainfalls from 0.4 to $1.4 \mathrm{~mm}$. They conclude that rain and fog cause the difference in link quality. On the other hand, they report a systematic change in signal strength every 24 hours which might indicate that the temperature plays a nonnegligible role. Capsuto et al. 4] analyze the effects of snow and ice on sensor networks using an outdoor deployment on a flat roof. They report $30 \mathrm{~dB}$ fades in their static deployment due to freezing rain and freezing fog.

In contrast to the findings above, Thelen et al. [6] have noticed that in a potato field deployment, radio waves propagate better during the night and during rain, i.e. in weather conditions with high humidity. They believe that humidity impacts the reflection coefficient of the top of the potato canopy. Also many radio vendors state that impact of rain on communication in the $2.4 \mathrm{GHz}$ band should be insignificant [7, 8, 9].

Bannister et al. have shown that high temperatures negatively affect communication between sensor nodes [1]. In their deployment in the Sonoran Desert of the southwestern United States, the reduction of the signal strength was largest during the hottest time of the day.

Given these discrepancies, we set up multiple experiments in order to quantify and compare the impacts of atmospheric events such as rain, snow, fog, and temperature. Our experimental results indicate that the changes in temperature can indeed affect radio communication much more than light rainfall or humidity. In addition to the experiments by Bannister et al., we measure the impact of low temperatures on radio communication, providing experimental results for two platforms at different radio frequencies. Similar to their experiments, we focus on the Received radio Signal Strength Indicator (RSSI), but we also measure and analyze the Link Quality Indicator (LQI) and noise floor.

Most sensor networks for industrial control and automation applications must comply with the ATEX 95 equipment directive 94/9/EC for equipment and protective systems intended for use in potentially explosive atmospheres. For example, the RUNES 11 technology roadmap for industrial control and automation highlights that all new networked embedded components should comply with the ATEX directive [5]. To the best of our knowledge, however, there are no studies that assess if compliance with this particular standard has an impact on wireless sensor networks performance. Our measurements aim to close this knowledge gap.

\footnotetext{
1 RUNES was an European Commission funded project (contract IST-004536).
} 


\section{Impact of temperature in outdoor deployments}

Sensor nodes communicate using low power, short range radio transceivers, such as the $2.4 \mathrm{GHz}$ Chipcon CC2420 [10] on the Tmote Sky platform [11] and the Chipcon $868 \mathrm{MHz}$ CC1020 [12] on the Scatterweb Modular Sensor Board (MSB430) [13, 14]. As other modern single-chip transceivers, both radio chips offer indicators to measure the link or signal quality, such as the Received Signal Strength Indicator (RSSI) and the Link Quality Indicator (LQI). Researchers have investigated the relationship between these indicators and the Packet Reception Rate (PRR) [15, 16, 17], showing that the indicators represent a good estimation of packet delivery.

Hardware components for outdoor deployments are usually designed for an industrial grade operating temperature range of $-40^{\circ} \mathrm{C}$ to $+85^{\circ} \mathrm{C}$, to ensure uninterrupted operation under various environmental conditions. Temperature changes, however, may cause crystal frequency to shift, thermal noise level of the transceiver to increase, and amplifiers to saturate [18], resulting in potential performance degradation of the radio device.

In order to quantify the impact of temperature on sensor nodes communication, we carry out different experiments with two different platforms. We want to assess the effects of both high and low temperatures on wireless communication since long-running outdoor deployments may be exposed to large temperature variations.

We further investigate the behavior of several hardware indicators, such as Received Signal Strength Indicator (RSSI), Link Quality Indicator (LQI), i.e. the correlation value of the first eight symbols of the received PHY header (CCI), and noise floor. We also verify that platforms using different radio frequencies show the same behavior.

\subsection{Experimental Setup}

We deploy three Tmote Sky [11] nodes in a wheat field in Govone, Italy during the end of December. We place the nodes 1,5 meters above ground, at a distance close to the border of their communication range. Nodes are supported by wood pieces fastened to the ground, to withstand wind blasts. The surface of the wheat field is flat, with no discrepancies. In our experiments, we set the transmission power to approximately $-5 \mathrm{dBm}$. Our nodes run the Contiki operating system [19]. Our deployment lasts seven consecutive days.

The sink node triggers a sender node to send 256 consecutive packets with a size of eight bytes on a specific channel. The sink node stores RSSI, LQI and noise floor readings for each received packet. It also stores environmental information such as temperature, humidity, sunlight and battery voltage of both nodes. In order to record the data without intrusion, the information is forwarded to a third node that acts as a data logger. We measure temperature and humidity using the on-board Sensirion SHT11 sensor [20]. To protect them from rain we keep the sensor nodes in plastic boxes 


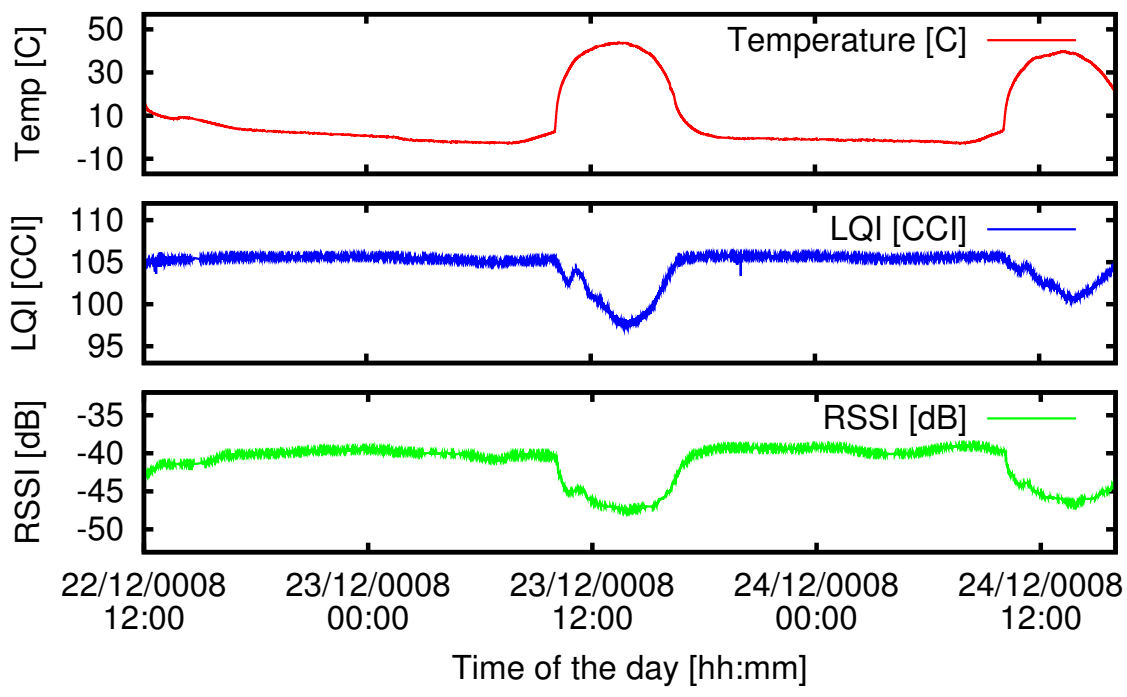

Fig. 1. Changes of RSSI and LQI of received packets follow the pattern of temperature changes: the higher the temperature, the worse the radio signal strength and the link quality. Data is retrieved in a 48-hour outdoor deployment in a wheat field in Govone, Italy.

We also carry out indoor experiments, in which we create a difference in temperature of more than $70^{\circ} \mathrm{C}$ on both Tmote Sky platforms and MSB430 nodes, by moving the nodes from a cold environment (refrigerator) to a warm environment and vice versa, while logging the data about the received packets. The position of the motes remain fixed, and there is no human interaction because the data is collected at a third node far away from the communicating ones. Temperature reading increases slowly, and should be accurate since the SHT11 sensor lies only few millimeters away from the radio chip. We also vary the temperature using a cooling spray that drops the temperature quickly to around $-35^{\circ} \mathrm{C}$. We obtain the same results which confirms the validity of our method. We set the transmission power to approximately $-25 \mathrm{dBm}$ in the Tmote Sky and to $-20 \mathrm{dBm}$ in the MSB430. We use the same software setup as in the previous experiment.

\subsection{Experimental Results}

Our outdoor and indoor experiments show the high impact of temperature on wireless connectivity between sensors, and confirm the experiments by Bannister et al. [1].

Our data show that temperature strongly affects the wireless communication on sensor networks platforms; in particular, the large difference in temperature 


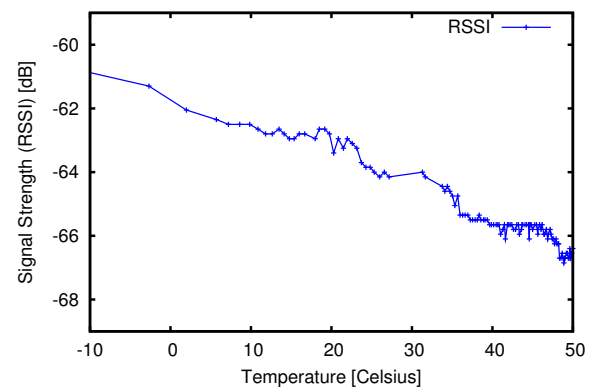

a) MSB430 platform

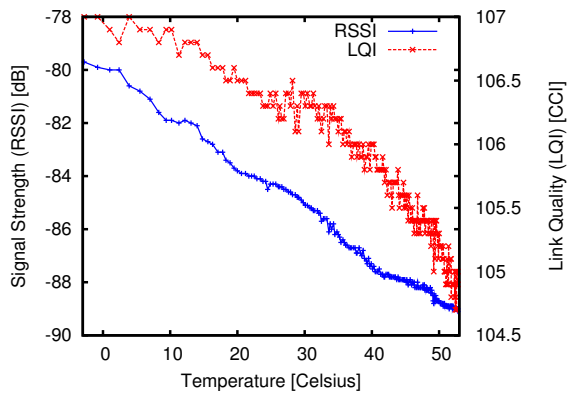

b) Tmote Sky platform

Fig. 2. Our indoor experiments confirm the results of our outdoor experiments. Both LQI and RSSI decrease as temperature increases. This applies not only to the Tmote Sky, but also to the MSB430 platform that uses a radio chip operating in a different frequency band.

between daytime and nighttime may affect the signal strength by several dBs. Figure 1 shows the data retrieved by the sink in the wheat field during 48 hours of sunny weather. Sensors experience large temperature shifts between day and night, amplified by the greenhouse effect caused by the plastic boxes covering the motes when the sun is shining. Figure 1 shows that the RSSI changes follow the same pattern as temperature: when the temperature increases, the signal strength decreases.

Our experimental results also show that LQI is affected by the temperature to a similar degree. Figure 1 shows that the higher the temperature, the worse the link quality. In the experiments, the motes are placed at close to the border of their transmission range. We carried out additional experiments to show that the significance of the impact on LQI decreases with distance. The temperature impact is considerable on weak links, whereas at short distances where LQI indicates a near-perfect link, the temperature effect is negligible.

The results of our indoor experiments depicted in Figure 2 confirm the results of the outdoor experiments. They reveal a similar behavior of the MSB430 platform that uses a radio chip operating in a different frequency band. The figure shows that the radio signal strength of received packets (RSSI) decreases monotonically as temperature increases. In summary, temperature variations affect communication between both MSB430 nodes and Tmote Sky nodes.

Figure $2 \mathrm{~b}$ further shows that also the Link Quality Indicator (LQI) is affected by the temperature. Since the LQI is not available in the CC1020 radio chip, it is not possible to measure it on the MSB430 platform. The indoor experiments with the Tmote Sky nodes, however, show how LQI and RSSI follow the same pattern of temperature, confirming the results of our outdoor experiments.

Figure 3 shows that, in addition to LQI and RSSI, noise floor readings are affected by temperature. As a direct consequence of the RSSI alteration, also the noise floor decreases as long as temperature increases. This is important since 


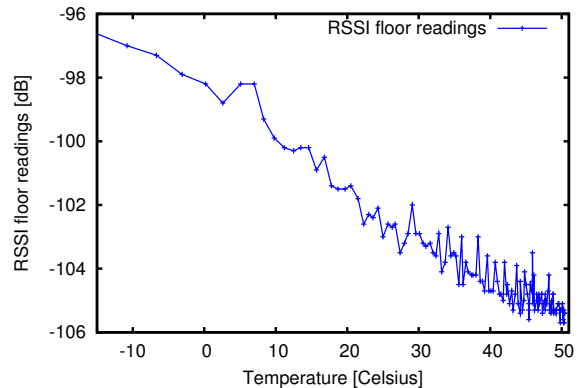

a) MSB430 platform

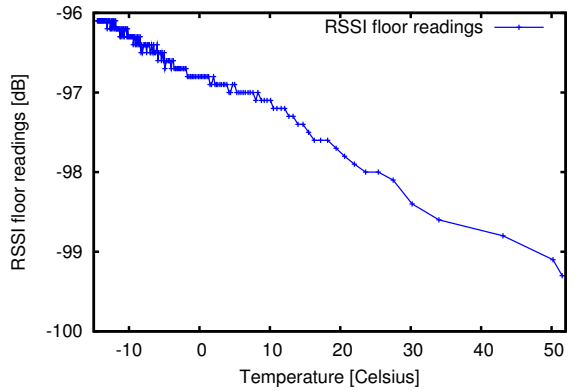

b) Tmote Sky platform

Fig. 3. Noise floor readings detected in our experiments carried out in different platforms at different temperatures.

the noise floor reading is often used to evaluate the interference and noise level in the communication [21, 22]. Figure 3 shows the impact of temperature on the noise floor readings on both the Tmote Sky and the MSB430 platforms.

\section{Impact of Humidity, Fog, Snow and Rainfall}

Not only temperature, but also other weather effects may impact the performance of outdoor deployments. As shown in Section 2 some researchers claim a strong impact of rain and other atmospherical events on radio signal strength. Others including radio manufacturers, however, indicate that the effects of rain on radio waves propagation are negligible at $2,4 \mathrm{GHz}$ frequencies $[6,7,8,9]$.

The presence of rain, fog, and snow may be strictly connected to a temperature change. Since the temperature variation may cause a loss in radio signal as we illustrated previously, it is important to discern the pure effect of temperature and atmospheric precipitations at the Line of Sight (LoS). Looking at the available data in published experiments, we can see that temperature changes may have influenced several deployments [3, 4], since there is a regular RSSI variation every 24 hours, even when there is no rain or fog.

During our outdoor deployment in the wheat field in Govone illustrated in Section 3.2, we report some light rainfall (around $1 \mathrm{~mm} /$ hour), snow and fog. Figure 4 shows the RSSI including the instants with fog, rain and snow. The radio communication is almost not affected by these atmospheric events, and no significant thermal variations are revealed in that period of time. In contrast, the impact of the temperature on LQI and RSSI is very high. Therefore, we design new experiments to distinguish the impact of temperature and weather on radio communication, trying to discern the temperature effect from the pure rainfall . 


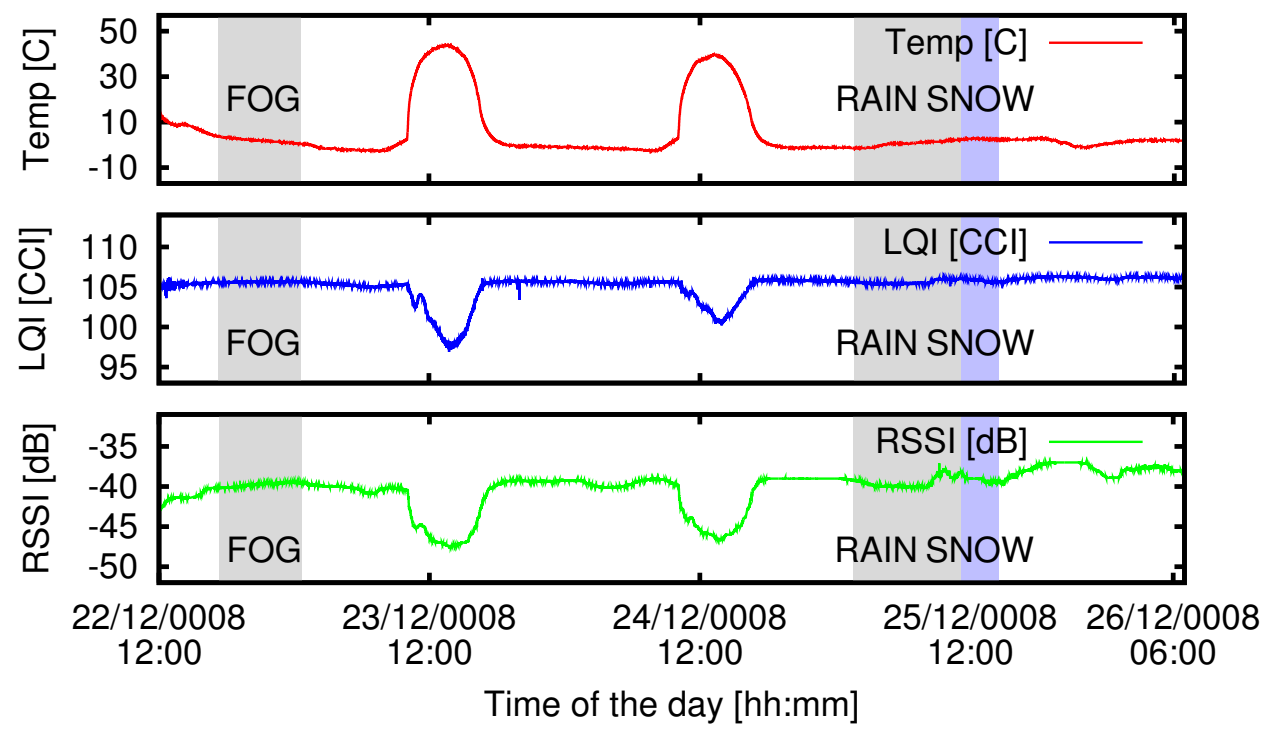

Fig. 4. Experimental data of the effects of temperature, fog, rain and snow on the radio signal strength. Data is retrieved in a wheat field during December in Govone $(\mathrm{CN})$, Italy.

\subsection{Experimental Setup}

In order to to distinguish between weather and temperature impact on wireless communication, we design new experiments. To avoid larger variations in temperature we place the nodes in two opposite office buildings in Kista, Sweden. The distance between the buildings is few dozens meters, and there are no obstacles. We deploy the motes at the fourth floor of the buildings. In this way, only rain and other environmental issues but not temperature affect the wireless communication. To easily detect weather impacts, we choose the transmission power so that the nodes can just communicate.

We take data about the amount of rain from a weather station situated $400 \mathrm{~m}$ away from the deployment area [23]. Data about rainfall, wind speed, temperature, humidity, solar light, snow and atmospheric pressure are available. In order to check the presence of fog, we use the images available from one of the webcams situated close to the deployment area. Figure 5 shows webcam images pointed towards the deployment area.

Our deployment also provides the possibility to compare the effects of microwaves and electromagnetic disturbances with temperature and weather effects. The buildings are offices, and thus during daytime, radio interference is higher than during the night. This implies that during working days, in office hours the signal strength may be varying more than usual. 


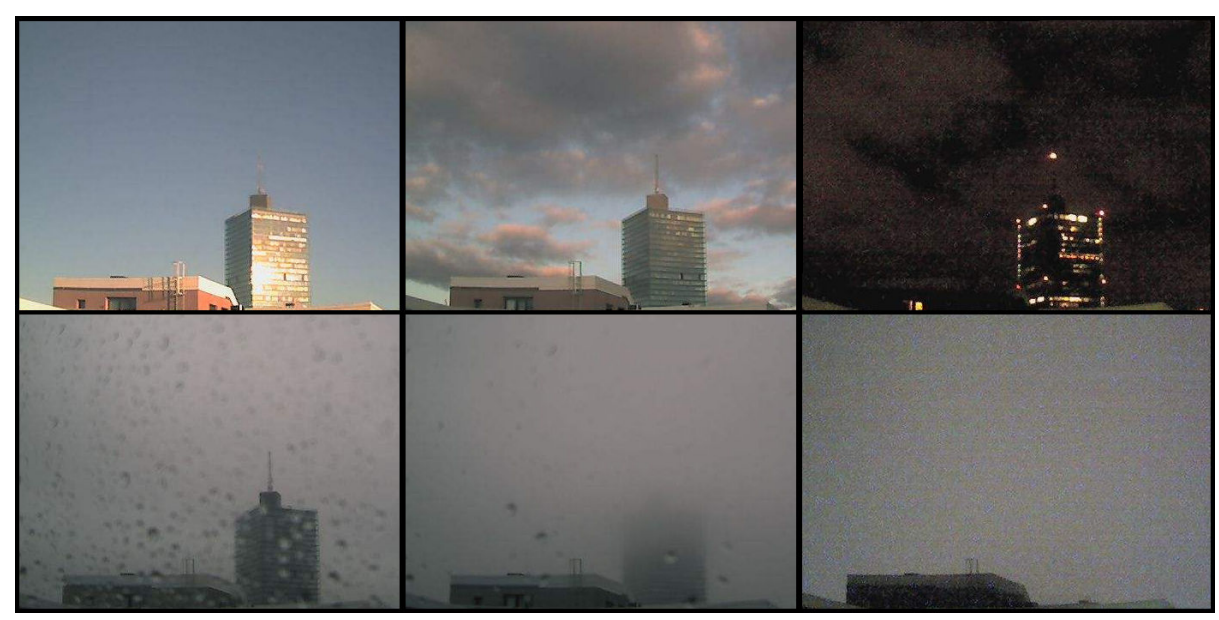

Fig. 5. Images captured from the webcam in order to check the presence of fog. From the bottom images it is possible to see both when rain and fog appeared in the deployed area. The figure appears under explicit permission of the owner [23].

\subsection{Experimental Results}

Between January and March 2009, we experienced several foggy days and many light and heavy rainfalls in Kista. We run the experimental setup explained in the previous section for 60 consecutive days. We combine the data from the sensor nodes and the information about the weather, isolating the instants of time with rain and fog.

Figure 6 illustrates the RSSI variation when there is fog in the deployment area. Although it is difficult to quantify the amount of fog, we consider respectively thin and thick fog the situations shown in the two latter pictures in Figure 5. Neither thin nor thick fog have a significant impact on the radio signal strength.

Figures 7 and 8 illustrate the RSSI variation in rainy days during January, February and March 2009 respectively. Rainfall is computed as the amount of rain fallen in the last 30 minutes. Although the rain intensity never reaches the possible values obtainable in tropical areas, the results indicate clearly that the impact of rain is less than the one caused by changes in temperature. In particular, when the rainfall amount is less than $1 \mathrm{~mm} / \mathrm{h}$, the effect of rain is almost negligible, while it increases with higher rain intensities. The only significant impact of rain we report is a significant packet loss rate and interruption of connectivity highlighted by a grey area in Figure 7. In this period, the rainfall reaches values much higher than the average intensity of $2 \mathrm{~mm} / \mathrm{h}$. In all other cases shown in Figures 7 and 8 , the RSSI variation is caused mainly by the noise generated by the office environment in which nodes are deployed rather than by weather effects.

Together with the results from our deployment in the wheat field, we can understand how the impact of temperature and electromagnetic disturbances is 

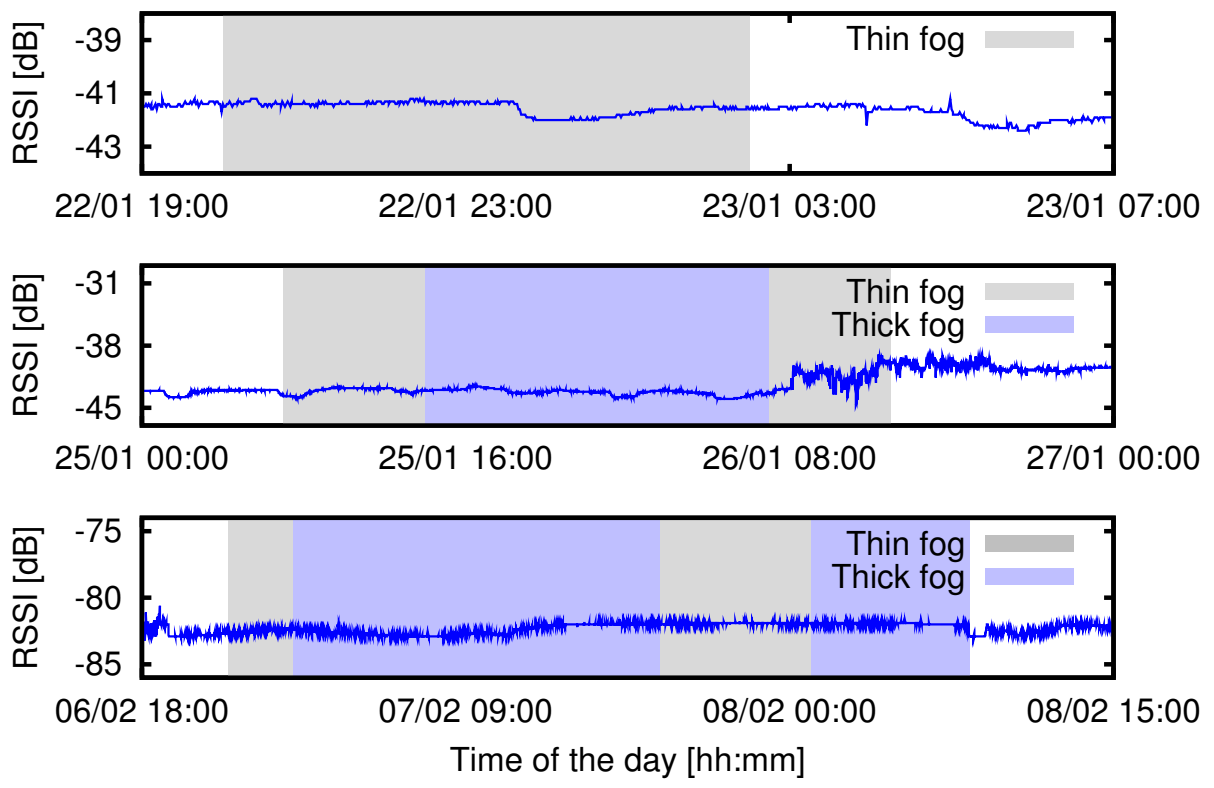

Fig. 6. Radio signal strength variation during foggy days in January and February in Kista, Sweden. The radio signal is neither affected by thin nor by thick fog.

much higher than the one caused by light rainfall or fog. However, really heavy rainfalls may affect the communication between nodes, especially if deployed at the border of their communication range.

\section{ATEX Compliance}

Many Industries have very specific requirements regarding the deployment of equipment within production environments. For example, in coal mines, chemical plants and oil refineries there are significant risks of fire or explosion due to flammable gas by-products released during production. Thus, wireless sensor nodes deployed in such an industrial context must adhere to specific standards. Within the European Union, regulations regarding the use of equipment in explosive atmospheres are known as the so called ATEX directives.

For an explosion to occur there is a requirement for a combustible material, oxygen and an ignition source. As it is very difficult to prevent the presence of combustible material and oxygen in large processing plants, explosion prevention concentrates on the ignition source. For example, it is almost impossible to guarantee that no oil spills occur in an oil refinery, where on the contrary it is much easier to secure electric systems to prevent sparks.

It is possible to obtain ATEX certification for a wireless sensor node. However, this is a costly and time consuming process which needs to be repeated whenever the sensor node is modified, for example, by adding new sensor arrays. 

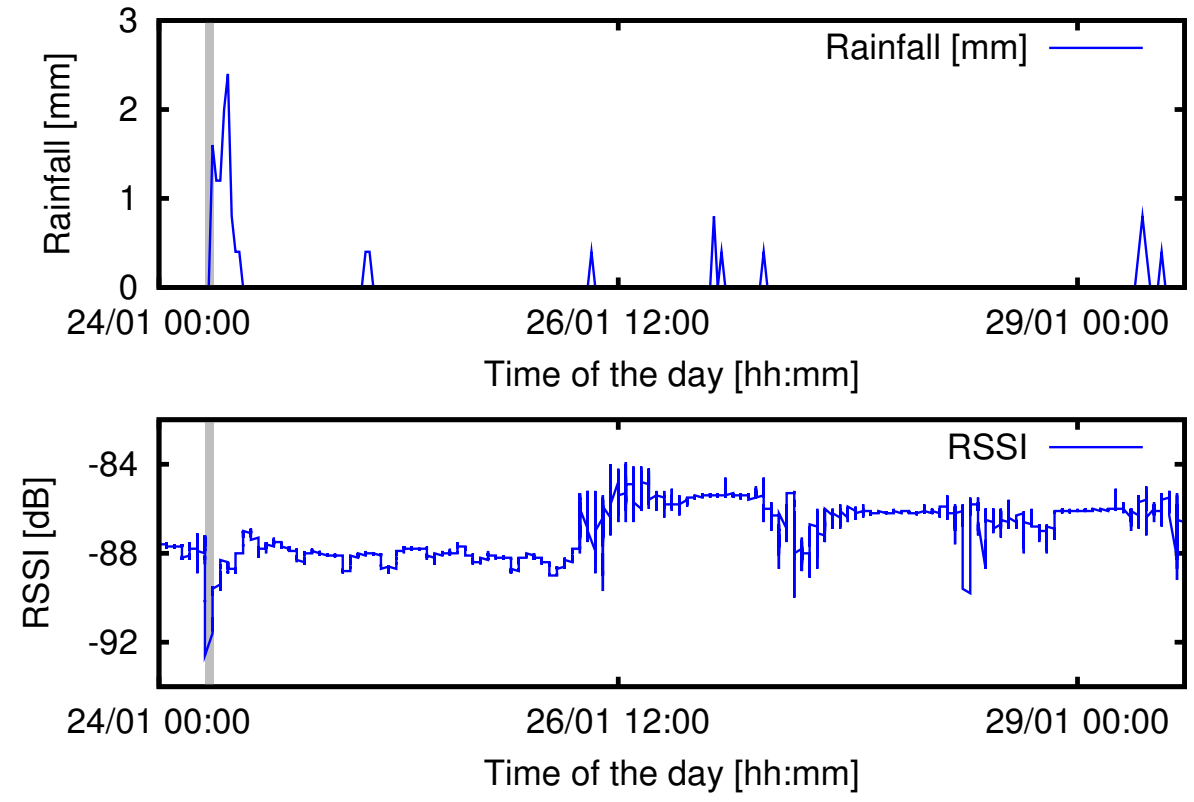

Fig. 7. Radio signal strength during rainy days in January in Kista, Sweden. The grey area highlights the instants in which we report a significant impact of rainfall on packet delivery. The RSSI variation is otherwised caused mainly by the electronic disturbances introduced by the office environment.
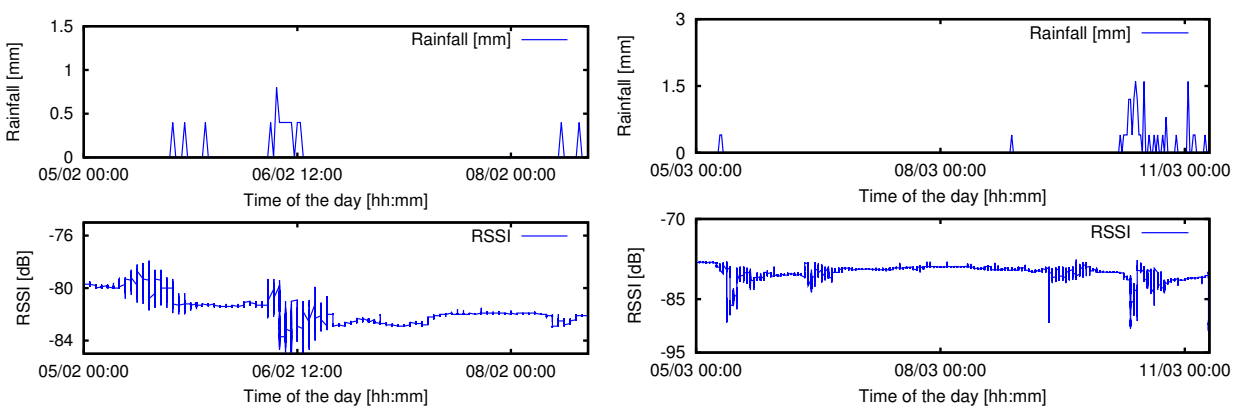

a) Rainy days in February

b) Rainy days in March

Fig. 8. Radio signal strength during rainy days in February and March in Kista, Sweden. The RSSI variation is caused mainly by the electronic disturbances introduced by the office environment rather than by the rainfall.

An alternative to obtaining ATEX certification for the sensor node is to obtain it for the enclosure that will house the node. Although certification of an enclosure is easier than that of a sensor node, this can also be avoided through the use of one of the many off the shelf ATEX-compliant enclosures that exist. Using an ATEX certified enclosure in this case means a standard sensor node can be 
used and alterations can be applied without re-running through the certification cycle.

Every element of a sensor node that is to be placed outside the ATEX certified box needs to be itself ATEX certified. For example, if the antenna of the node is placed outside the box it needs a certification on its own and has to be fitted with ATEX certified connectors to the main box. Hence it is highly desirable to keep all components such as antenna inside the box.

As other work has shown, placing nodes with antennas inside an enclosure can substantially affect the communication patterns. The effects that such encloses have can be reduce through design and the selection of material used in their construction. For example in a car-park application [24] a box was specially designed that minimizes the impact on communication. For the described industrial scenarios it is not possible to change the material of the ATEX box without potentially losing its protective properties.

Before such enclosures can be used for the above industrial applications, an investigation of how these might impact the communication capabilities of a node must take place.

\subsection{Experimental Setup}

We investigated the effects that ATEX casing have on node communications by conducting three experiments. The first experiment quantifies the effects that ATEX casing has on two communicating nodes at various distances and orientations. The second experiment was determines the potential communication distance of nodes with ATEX casings. The third experiment is conducted to discover the effects that the internal enclosure temperature has on communications. All experiments were carried out on Tmote Sky [11] nodes, which were placed into two separate ATEX compliant enclosures as shown in Figure 9. One node is used as a sender and the other as a receiver.

Experiment 1 The first experiment is carried out in-doors within a large empty meeting room with no obstacles or visible interference. Both nodes are placed one meter above the ground with a distance of $D$. The distance $D$ between sender and receiver is one of the variables in the experiments and is set at $5 \mathrm{~m}$, $10 \mathrm{~m}$ and $20 \mathrm{~m}$. For each communication test at distance $D$ the sender is used to transmit blocks of $i=256$ packets over a four second window. The sender's transmission power is set to $-5 \mathrm{dBm}$. For each communication test at distance $D$, four receiver orientations $\alpha$ were used $\left(\alpha=0^{\circ}, \alpha=90^{\circ}, \alpha=180^{\circ}, \alpha=270^{\circ}\right)$. The orientation of the transmitter is not changed. At the starting point $\alpha=0^{\circ}$ the sender and receiver antenna point towards each other. For each setting 10 transmission blocks are sent to the receiver which forwarded statistics to a third node connected to a laptop.

For each setting two experiments are conducted: one whilst the two nodes are cased in the ATEX compliant enclosures and one where the nodes are not encased. The average RSSI $R$ in dependence of the communication distance $D$ 


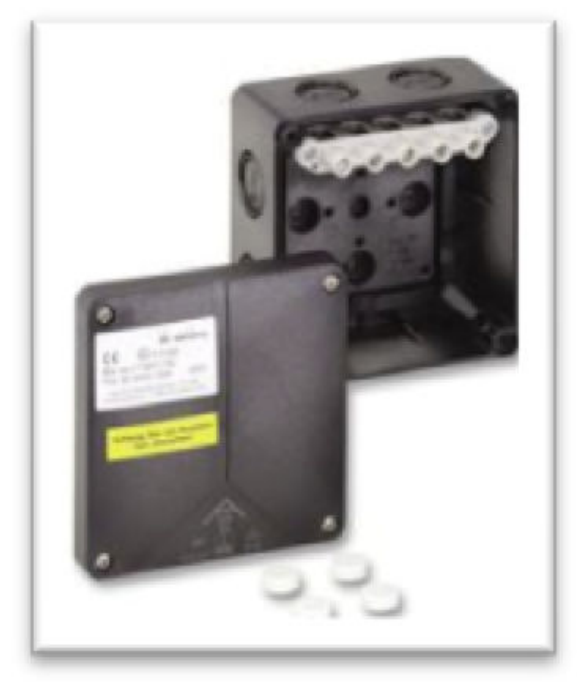

Fig. 9. Zone 2 ATEX compliant case.

and orientation $\alpha$ is determined. The results for this experiment are show in Figure 10 .

Experiment 2 The second experiment is carried out in a open field. The days prior to the experiment, it was raining, thus the chosen field is extremely wet. The field is not completely plane, a ditch of about $1 \mathrm{~m}$ is present (The impact of such small topology imperfections is discussed later with the results). The distance $D$ is increased between each test run in $10 \mathrm{~m}$ steps from $D=10 \mathrm{~m}$ to $D=170 m$ inclusive.

At each distance, $D, i=100$ packets with inter-arrival time of $100 \mathrm{~ms}$ are transmitted from the sender to receiver. The average RSSI $R$ in dependence of the communication distance $D$ is determined. For this test, sender and receiver are placed in ATEX casings with orientation $\alpha=0^{\circ}$. The results of this experiment are shown in Figure 11.

Experiment 3 The third experiment is carried out indoors within an office building environment. The distance is fixed at $D=5 \mathrm{~m}$. Three nodes are used similarly to experiment one, one transmitter, one receiver and one node to collect statistics. To fully ascertain the effects of temperature three different test settings are investigated in which the transmitter temperature $T_{s}$ and/or the receiver temperature $T_{r}$ is altered. In the first test the temperature of the sender is fixed at room temperature of $T_{s}=25^{\circ} \mathrm{C}$ and temperature of the receiver is slowly lowered from $\mathrm{Tr}=45^{\circ} \mathrm{C}$ to $\mathrm{Tr}=-15^{\circ} \mathrm{C}$. In the second test the temperature of the receiver is fixed at $T_{r}=25^{\circ} \mathrm{C}$ and the temperature of the sender is slowly lowered from $T_{s}=45^{\circ} \mathrm{C}$ to $T_{s}=0^{\circ} \mathrm{C}$. Finally during the last test the temperature of both the receiver and sender is raised and then slowly cooled to 
a temperature of $T_{r}=T_{s}=0^{\circ} \mathrm{C}$. The average RSSI $R$ in dependence of the transmitter temperature $T_{s}$ and the receiver temperature $T_{r}$ is determined. The results of this experiment are shown in Figure 12 to Figure 14.

\subsection{Results}

Experiment 1 The measured RSSI at the receiver is found to be affected by both node orientation and ATEX Casing. Figure 10 shows at the $5 m$ distance point, the measurements taken whilst the devices are within the case is significantly better than those taken from the devices outside of the case. This changes as the devices moved further apart, where at the $10 \mathrm{~m}$ point there is a negligible difference between devices within the case to those outside of the case. At $20 \mathrm{~m}$ the devices outside the case out performed those within the case. Looking at the effects of orientation, no trends could be established to how orientation affects the measured RSSI at the receiver.

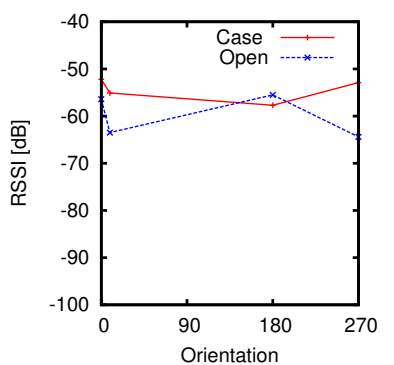

a) $D=5 m$

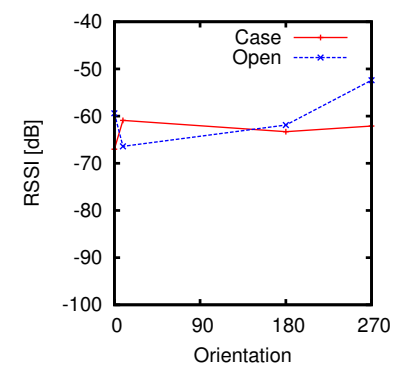

b) $D=10 \mathrm{~m}$

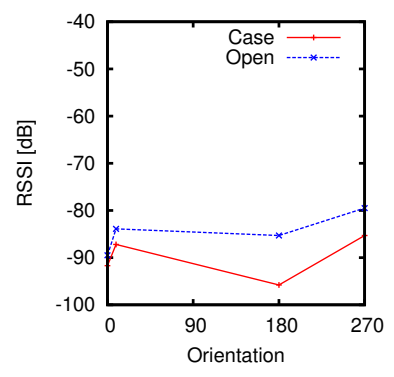

c) $D=20 \mathrm{~m}$

Fig. 10. Effects of ATEX compliant casing on RSSI in with different configurations.

Experiment 2 Figure 11 shows that with increasing distance the RSSI value drops. However, from a distance of approximately $70 m$ to $80 m$ meters, the RSSI is stable at a low value. This can be attributed to the fact that the RSSI is only recorded when a packet is received at the receiver, therefore when packet is lost, which occurs as the distance is increased and signal degrades, less RSSI sample points are measured. At the $90 \mathrm{~m}$ point packet loss does increase, as obstacles in the field are encounter particularly the dip in RSSI at around $100 \mathrm{~m}$ can be attributed to the fact that a small water filled ditch was present at this distance. Obviously such small obstacles have a significant impact on the communication patterns. The maximum communication range during the experiment with ATEX casings is found to be approximately $140 \mathrm{~m}$. From this distance on significant losses are recorded. However, an abnormality occurred at $160 \mathrm{~m}$ where an isolated patch with good communication conditions is found. 


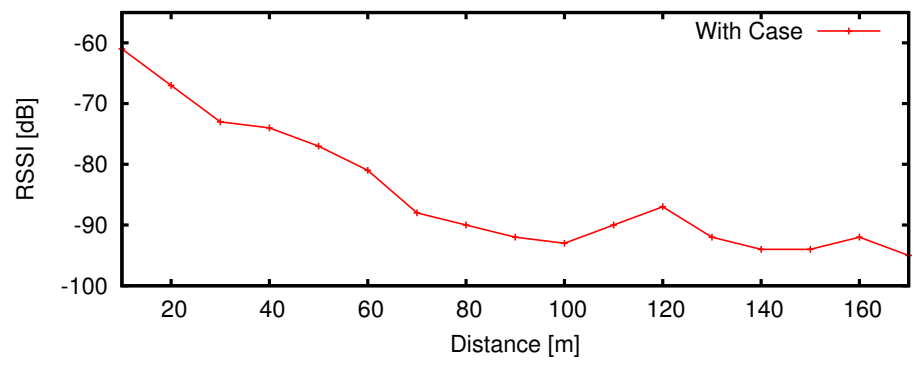

Fig. 11. Effects of ATEX compliant casing on RSSI over distance.

Experiment 3 A high temperature of either the receiver or transmitter has a worsening effect on the recorded RSSI. When the receiver is cooled from $45^{\circ} \mathrm{C}$ to approximately $-10^{\circ} \mathrm{C}$, RSSI rises by approximately $5 \mathrm{~dB}$. Likewise when the temperature of the transmitting device is cooled from $45^{\circ} \mathrm{C}$ to $0^{\circ} \mathrm{C}$, RSSI rises again by $5 d B$. It is also found that when the temperature of both the sender and receiver is cooled, there effects are summed to $10 \mathrm{~dB}$.
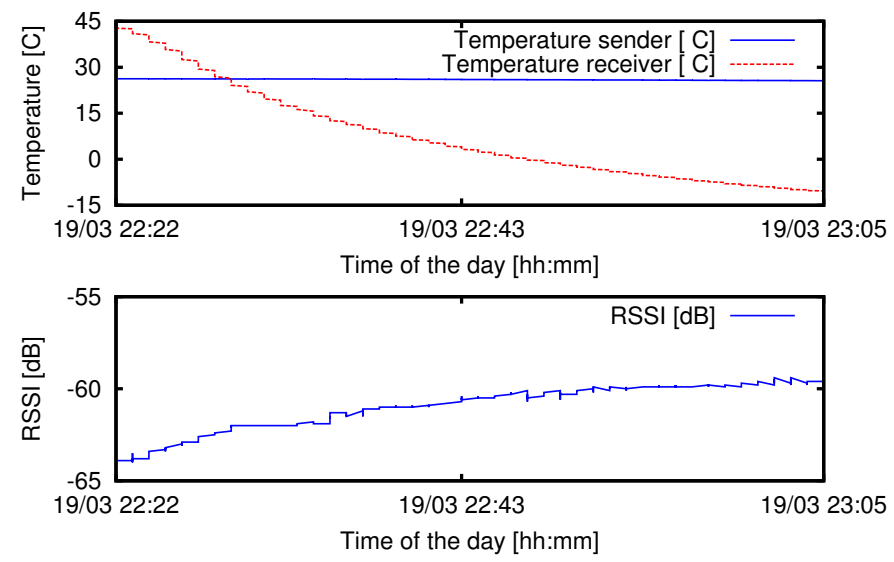

Fig. 12. Effects of the temperature on communication when the ATEX cases of only the sender is cooled.

\subsection{Findings}

During our experimentation with ATEX compliant casing we found that the effects on communication were negligible at distances of approximately $10 \mathrm{~m}$. As this point is passed, RSSI falls as distance between receiver and transmitter increases. Conversely the opposite occurs as the communicating nodes are brought closer together. Furthermore it was found that node configuration has a major role in communication, simply turning the node in a single plane in some 

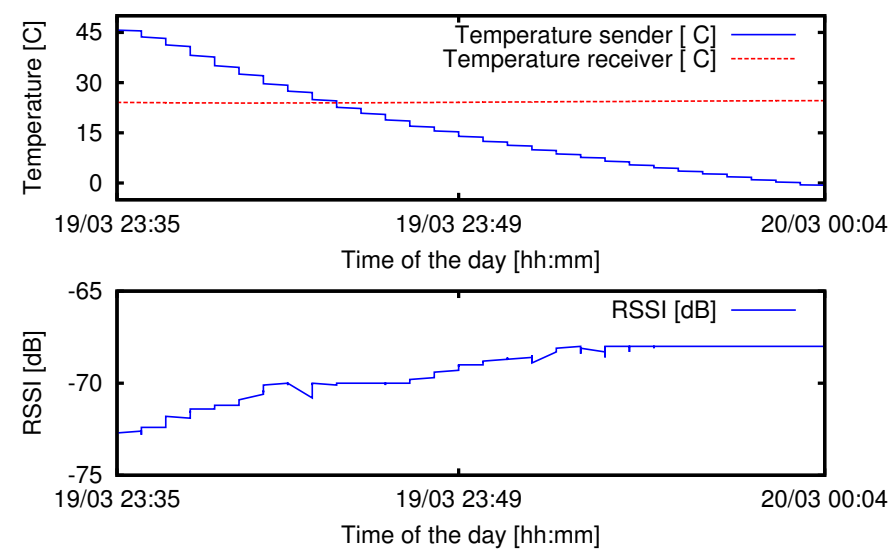

Fig. 13. Effects of the temperature on communication when the ATEX cases of only the receiver is cooled.
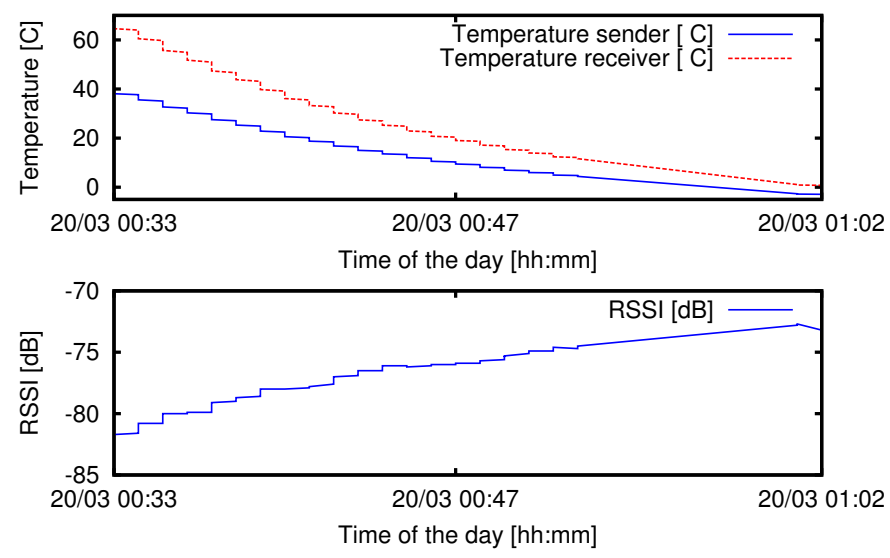

Fig. 14. Effects of the temperature on communication when the ATEX cases of both receiver and sender are cooled.

cases can reduce RSSI by $10 d B$ much more than the measured effects of ATEX enclosures.

A typical maximum range of nodes communication from inside separate ATEX enclosures is measured to be approximately $140 \mathrm{~m}$ before substantial signal degradation. As we show in this paper this value is very easily influenced by temperature or orientation so is here purely to depict a possible communication range in one environment. Similarly to the findings in the early sections of this paper, it is discovered that raising the temperature of the node within an ATEX enclosure has a negative effect on communication. Not only is the temperature of the receiver important but also that of the sender. 


\section{Summary and Conclusions}

Several industrial deployments require performance assurances. Outdoor deployments are exposed to varying environmental conditions, in particular rain, fog, and temperature, which might have an impact on the wireless communication between the deployed sensor nodes. Furthermore, in potentially explosive environments, it is necessary to enclose the sensor nodes in ATEX compliant enclosures. In order to evaluate the impact of the environmental conditions and ATEX-compliant cases, we perform multiple experiments with real hardware. We show that the temperature has the largest impact on communication. The impact of fog and rain is not severe until the rain is heavy, i.e. more than 2$3 \mathrm{~mm} /$ hour. The impact of ATEX-compliant enclosures on communication is small.

Our results suggest that when deploying an outdoor industrial sensor network, the current temperature needs to be taken into account. The distance between the nodes should not be close to the maximum transmission range, in particular when the deployment is done during the colder time of the year. Our results also suggest that through a careful planning and deployment, that takes into account the environmental conditions and the potential impact on temperature and rain, it is possible to provide reliable wireless sensor communication.

\section{Acknowledgments.}

The research leading to these results has received part-funding from the European Community's Seventh Framework Programme (FP7/2007-2013) under grant agreement number 224282.

Part of this work has been performed within the SICS Center for Networked Systems funded by VINNOVA, SSF, KKS, ABB, Ericsson, Saab Systems, TeliaSonera and T2Data. This work has been partially supported by CONET, the Cooperating Objects Network of Excellence, funded by the European Commission under FP7 with contract number FP7-2007-2-224053.

\section{References}

1. Kenneth Bannister, Gianni Giorgetti, and Sandeep K.S. Gupta. Wireless sensor networking for hot applications: Effects of temperature on signal strength, data collection and localization. In Proceedings of the fifth Workshop on Embedded Networked Sensors (HotEmNets'08), Charlottesville, Virginia, USA, June 2008.

2. Giuseppe Anastasi, Alessio Falchi, Andrea Passarella, Marco Conti, and Enrico Gregori. Performance measurements of motes sensor networks. In Proceedings of the 7th ACM international symposium on Modeling, analysis and simulation of wireless and mobile systems (MSWiM'04), Venice, Italy, October 2004.

3. Jingbo Sun and Rachel Cardell Oliver. An experimental evaluation of temporal characteristics of communication links in outdoor sensor networks. In Proceedings of the second Workshop on Real-World Wireless Sensor Networks (REALWSN'06), Uppsala, Sweden, June 2006. 
4. Benji Capsuto and Jeff Frolik. Demo abstract: A system to monitor signal fade due to weather phenomena for outdoor sensor systems. In Proceedings of the Fifth International Conference on Information Processing in Sensor Networks (IPSN'06), Nashville, TN, USA, April 2006.

5. ATEX Guidelines. Web page, http://ec.europa.eu/enterprise/atex/guide/. Visited 2009-02-14.

6. John Thelen, Daan Goense, and Koen Langendoen. Radio wave propagation in potato fields. In Proceedings of the 1st workshop on wireless network measurement, Riva del Garda, Italy, April 2005.

7. CWNP certifications. Rain fade margin. Web site. http://www.cwnp.com. Visited 2009-02-14.

8. Covad Communications Group. Wireless networking backgrounder. Web site. http://www.covadwireless.com/documents/wirelessWhitepaper.pdf, March 2007.

9. AFAR Communications Inc. $900 \mathrm{mhz}$ versus $2.4 \mathrm{ghz}$ in long distance links. Web site, http://www.afar.net/tutorials/900-mhz-versus-2.4-ghz/. Visited 2009-02-14.

10. Chipcon AS. CC2420 datasheet - 2.4 GHz IEEE 802.15.4 / ZigBee-Ready RF Transceiver (Rev. B), March 2007.

11. Moteiv Corporation. Tmote Sky - Datasheet, edition 1.04 edition, November 2006.

12. Chipcon AS. CC1020 datasheet - Low-Power RF Transceiver for Narrowband Systems (Rev. B), July 2008.

13. ScatterWeb GmbH. MSB: modular sensor board, version 1.0 edition. Visited 200902-14.

14. Michael Baar, Enrico Köppe, Achim Liers, and Jochen Schiller. Poster abstract: The scatterweb msb-430 platform for wireless sensor networks. In Contiki Workshop '07, Kista, Stockholm, Sweden, March 2007.

15. Kannan Srinivasan and Philip Levis. Rssi is under appreciated. In Proceedings of the Third Workshop on Embedded Networked Sensors (EmNets'06), Cambridge, MA, USA, May 2006.

16. Kannan Srinivasan, Prabal Dutta, Arsalan Tavakoli, and Philip Levis. Understanding the causes of packet delivery success and failure in dense wireless sensor networks. In Proceedings of the 4th ACM Conference on Embedded Networked Sensor Systems (SenSys'06), pages 419-420, Boulder, Colorado, USA, November 2006.

17. Matthew M. Holland, Ryan G. Aures, and Wendi B. Heinzelman. Experimental investigation of radio performance in wireless sensor networks. In Proceedings of the 2nd IEEE Workshop on Wireless Mesh Networks (WiMesh'06), Reston, Virginia, USA, September 2006.

18. Chipcon AS. CC2400 datasheet - 2.4 GHz Low-Power RF Transceiver (Rev. 1.5), March 2006. http://focus.ti.com/lit/ds/symlink/cc2400.pdf - Last visited January 2009.

19. Adam Dunkels, Björn Grönvall, and Thiemo Voigt. Contiki - a lightweight and flexible operating system for tiny networked sensors. In Workshop on Embedded Networked Sensors, Tampa, Florida, USA, November 2004.

20. Sensirion AG. SHT1x Humidity and Temperature Sensor datasheet, version 2.04 edition, May 2005.

21. Dhananjay Lal, Arati Manjeshwar, Falk Herrmann, Elif Uysal-Biyikoglu, and Abtin Keshavarzian. Measurement and characterization of link quality metrics in energy constrained wireless sensor networks. In IEEE Global Telecommunications Conference (GLOBECOM '03), pages 446-452, December 2003. 
22. M. Senel, K. Chintalapudi, Dhananjay Lal, A. Keshavarzian, and E.J. Coyle. A kalman filter based link quality estimation scheme for wireless sensor networks. In IEEE Global Telecommunications Conference (GLOBECOM '07), pages 875-880, November 2007.

23. Bengt Fogelberg. Kistavädret. Web site. http://soloregn.se/. Visited 2009-02-14.

24. Jonathan P. Benson, Tony O'Donovan, Padraig O'Sullivan, Utz Roedig, Cormac J. Sreenan, John Barton, Aoife Murphy, and Brendan O'Flynn. Car-park management using wireless sensor networks. In $L C N$, pages 588-595, 2006. 